\title{
Cardiac dose reduction with deep inspiration breath hold for left-sided breast cancer radiotherapy patients with and without regional nodal irradiation
}

\author{
Rosanna Yeung ${ }^{1}$, Leigh Conroy ${ }^{2,3}$, Karen Long ${ }^{4}$, Daphne Walrath ${ }^{4}$, Haocheng Li ${ }^{5}$, Wendy Smith ${ }^{2,3,6}$,
} Alana Hudson ${ }^{2,6}$ and Tien Phan ${ }^{1 *}$

\begin{abstract}
Background: Deep inspiration breath hold (DIBH) reduces heart and left anterior descending artery (LAD) dose during left-sided breast radiation therapy (RT); however there is limited information about which patients derive the most benefit from DIBH. The primary objective of this study was to determine which patients benefit the most from DIBH by comparing percent reduction in mean cardiac dose conferred by DIBH for patients treated with whole breast RT \pm boost (WBRT) versus those receiving breast/chest wall plus regional nodal irradiation, including internal mammary chain (IMC) nodes (B/CWRT + RNI) using a modified wide tangent technique. A secondary objective was to determine if DIBH was required to meet a proposed heart dose constraint of $D_{\text {mean }}<4 \mathrm{~Gy}$ in these two cohorts.

Methods: Twenty consecutive patients underwent $C T$ simulation both free breathing (FB) and DIBH. Patients were grouped into two cohorts: WBRT $(n=11)$ and B/CWRT + RNI $(n=9)$. 3D-conformal plans were developed and FB was compared to DIBH for each cohort using Wilcoxon signed-rank tests for continuous variables and McNemar's test for discrete variables. The percent relative reduction conferred by DIBH in mean heart and LAD dose, as well as lung $V_{20}$ were compared between the two cohorts using Wilcox rank-sum testing. The significance level was set at 0.05 with Bonferroni correction for multiple testing.

Results: All patients had comparable target coverage on DIBH and FB. DIBH statistically significantly reduced mean heart and LAD dose for both cohorts. Percent reduction in mean heart and LAD dose with DIBH was significantly larger in the B/CWRT + RNI cohort compared to WBRT group (relative reduction in mean heart and LAD dose: $55.9 \%$ and $72.1 \%$ versus $29.2 \%$ and $43.5 \%, p<0.02$ ). All patients in the WBRT group and five patients (56\%) in the B/ CWBRT + RNI group met heart $\mathrm{D}_{\text {mean }}<4$ Gy with FB. All patients met this constraint with DIBH.

Conclusions: All patients receiving WBRT met $\mathrm{D}_{\text {mean }}$ Heart $<4$ Gy on FB, while only slightly over half of patients receiving $B / C W R T+R N I$ were able to meet this constraint in $F B$. DIBH allowed a greater reduction in mean heart and $L A D$ dose in patients receiving B/CWRT + RNI, including IMC nodes than patients receiving WBRT. These findings suggest greatest benefit from DIBH treatment for patients receiving regional nodal irradiation.
\end{abstract}

Keywords: Breast cancer, Deep inspiration breath hold, Dosimetry, Regional nodal irradiation, Patient selection, Cardiac dose

\footnotetext{
* Correspondence: Tien.Phan@albertahealthservices.ca

${ }^{1}$ Division of Radiation Oncology, Department of Oncology, University of

Calgary, 2500 University Dr NW, Calgary, Alberta T2N 1 N4, Canada

Full list of author information is available at the end of the article
}

(c) 2015 Yeung et al. Open Access This article is distributed under the terms of the Creative Commons Attribution 4.0 International License (http://creativecommons.org/licenses/by/4.0/, which permits unrestricted use, distribution, and reproduction in any medium, provided you give appropriate credit to the original author(s) and the source, provide a link to the Creative Commons license, and indicate if changes were made. The Creative Commons Public Domain Dedication waiver (http://creativecommons.org/publicdomain/zero/1.0/) applies to the data made available in this article, unless otherwise stated. 


\section{Background}

Adjuvant radiation therapy (RT) for breast cancer patients reduces the risk of local recurrence and improves overall survival $[1,2]$. RT to the left breast and chest wall results in non-negligible dose to the heart and coronary arteries. Several large studies have demonstrated increased cardiac mortality associated with RT for left-sided breast cancer [3-5]. The use of cardiotoxic chemotherapy may further increase this risk [6].

Techniques to minimize irradiation of cardiac structures without compromise to target coverage have been developed including the use of deep inspiration breath hold (DIBH). While there is not yet any clinical evidence showing reduced cardiac toxicity or morbidity when using DIBH, numerous planning studies have demonstrated decreased dose to cardiac structures when compared to FB [7-15]. However, threshold doses to the heart and coronary arteries have not been determined [13]. A recent international breast cancer randomized trial (NSABP B51/RTOG1304), has proposed that the mean heart dose ( $D_{\text {mean }}$ Heart) should be $<4$ Gy during left-sided breast/ chest wall irradiation [16]. Dose limits have not been established for the left-anterior descending coronary artery (LAD), which may be the crucial target to mitigate ischemic heart disease risk from RT $[17,18]$.

DIBH is not uniformly implemented. A survey conducted by the authors in May 2014 identified that only four of fourteen cancer centers in Western Canada were using DIBH during RT for left-sided breast cancer, in part because of concerns about technical complexity and infrastructure constraints. Our institution implemented a visually monitored DIBH technique in June 2013 to avoid challenges with limited access to respiratory gating devices on older linear accelerators and to reduce simulation and treatment delivery time for DIBH.

The primary objective of this study was to identify which patients would benefit most from DIBH by determining whether a difference in percent mean cardiac dose reduction exists in patients receiving whole breast radiotherapy alone compared to those also receiving regional nodal irradiation (RNI), including internal mammary chain (IMC) nodal RT using a modified wide tangent technique. Our secondary objective was to determine if DIBH was required to meet the proposed mean heart dose constraint of $<4$ Gy.

\section{Methods}

\section{Patient population}

Twenty consecutive patients with left-sided breast cancer treated at our center starting in June 2013 were included if they were able to maintain $\mathrm{DIBH} \geq 20$ seconds. All patients received CT simulation with standard FB and DIBH and were treated on a wing-board. The DIBH technique involved training patients immediately prior to CT simulation. The distances from table-top to the mid-axillary line in FB and DIBH were recorded and displacements were calculated. Once reproducibility in DIBH was established based on these measurements, patients were translated through the CT with bellows (Philips, The Netherlands) to ensure stability of breath hold.

Clinical target volumes (CTV) included the breast or chest wall $\left(\mathrm{CTV}_{\mathrm{b}}\right)$ for all patients. If the regional nodes were to be treated, additional CTVs for the internal mammary chain nodes inclusive of the first to third interspaces $\left(\mathrm{CTV}_{\mathrm{IMC}}\right)$ and axillary plus supraclavicular nodes were delineated. $C T V_{b}$ was based on RTOG guidelines [19]. IMC nodes were delineated by a $1 \mathrm{~cm}$ diameter circle around the internal mammary vessels from the first to third intercostal space, and cropped from lung and bone [20]. If boost to the seroma was deemed necessary, $\mathrm{PTV}_{\text {boost }}$ was delineated by expanding the seroma contour by $1 \mathrm{~cm}$ in all directions. Organs at risk (OAR) were defined as the heart, LAD, and left lung. The heart and LAD were contoured based on previously published guidelines [21].

\section{Treatment planning}

For each patient, 3D-CRT plans on the FB and DIBH scans were developed using forward planning, field-based techniques. Tangential medio-lateral opposed fields with dynamic wedges were used to cover the $\mathrm{CTV}_{\mathrm{b}} \pm \mathrm{CTV}_{\mathrm{IMC}}$. IMC nodes were covered using a modified wide tangent technique [20] where tangent fields were widened to include the $C T V_{\text {IMC }}$ and narrowed inferiorly to reduce lung and cardiac doses. Supraclavicular and axillary nodes were covered by a single anterior field, or opposed anteriorposterior fields as determined by the treating radiation oncologist. The aim of the plans was to cover $\geq 95 \%$ of the $\mathrm{CTV}_{\mathrm{b}} \pm$ supraclavicular/axillary nodes with $\geq 95 \%$ of the prescription dose. If $\mathrm{CTV}_{\mathrm{IMC}}$ was included, target coverage of $\geq 80 \%$ of the prescription dose to $100 \%$ of the volume was used.

\section{Dosimetric evaluation}

$\mathrm{CTV}_{\mathrm{b}}$ volumes, $\mathrm{D}_{95}$ coverage of the $\mathrm{CTV}_{\mathrm{b}}$, and IMC coverage (if treated) on FB and DIBH scans for each patient were recorded. Heart $\mathrm{V}_{25}$, left lung $\mathrm{V}_{20}$, and mean doses $\left(D_{\text {mean }}\right)$ to the heart and LAD on FB and DIBH scans were determined. Percent dose reduction conferred by DIBH was calculated for mean heart and mean LAD doses as well as left lung $V_{20}$ for each patient.

\section{Statistical analysis}

Patients were classified into two treatment cohorts: patients who received whole breast radiotherapy alone \pm boost (WBRT), and patients who received breast or chest wall radiotherapy plus regional nodal irradiation, including the IMC and supraclavicular nodes (B/CWRT + RNI). Results were analyzed using $\mathrm{R}$ Software version 3.1.2 [22]. To evaluate FB vs. DIBH within each cohort, Wilcoxon 
signed-rank tests were used for continuous variables and McNemar's test was used to test differences for discrete variables. The percent relative reduction when using DIBH as opposed to FB was compared between the two cohorts for mean heart dose, mean LAD dose, and lung $\mathrm{V}_{20}$ using Wilcoxon rank-sum tests. The Bonferroni correction method was used to correct for multiple comparisons. The initial significance level of 0.05 was divided by the number of tests to obtain a modified significance level.

\section{Results}

\section{Demographics}

Eleven patients received WBRT and nine patients received B/CWRT + RNI. Table 1 shows the subjects' baseline demographics and radiotherapy treatment characteristics. Stage distribution dictated the treatment technique and therefore, was different between cohorts. Dose and fractionation prescribed in this study represents standard treatment prescriptions at our institution. The most common WBRT dose fraction was 42.5 Gy in 16 fractions. For

Table 1 Baseline demographic and radiotherapy treatment parameters for left-sided breast cancer patients by treatment cohort

\begin{tabular}{lll}
\hline Characteristics & $\begin{array}{l}\text { WBRT } \\
(n=11)(\%)\end{array}$ & $\begin{array}{l}\text { B/CWRT }+ \text { RNI } \\
(n=9)(\%)\end{array}$ \\
\hline Median age (years), range & $47(39-54)$ & $51(34-69)$ \\
AJCC Stage & & \\
DCIS & $3(27)$ & $0(0)$ \\
I & $5(45)$ & $0(0)$ \\
II & $3(27)$ & $5(55)$ \\
III & $0(0)$ & $4(44)$ \\
ER/PR positive (for invasive disease) & $7 / 8(88)$ & $7 / 9(78)$ \\
HER 2+ (for invasive disease) & $2 / 8(25)$ & $2 / 9(22)$ \\
Surgery & & \\
Breast conserving & $11(100)$ & $5(56)$ \\
Mastectomy & $0(0)$ & $4(44)$ \\
RT boost to seroma & $4(36)$ & $2(22)$ \\
RT to internal mammary nodes & $0(0)$ & $9(100)$ \\
Breast/Chest wall RT dose & & \\
(Gy/\# fraction) & & $2(67)$ \\
$40 / 16$ & & \\
$42.5 / 16$ & N/A (0) & \\
$45 / 25$ & $10(91)$ & $4(44)$ \\
$50 / 25$ & $0(0)$ & $1(11)$ \\
Supraclavicular nodal RT dose & $1(9)$ & $2(22)$ \\
(Gy/\# fraction) & & \\
$37.5 / 16$ & & \\
$45 / 25$ & & \\
\hline
\end{tabular}

patients receiving additional RNI, dose fractionation ranged from $40-50$ Gy in $16-25$ fractions to the breast or chest wall and 37.5 Gy-45 Gy in 16-25 fractions to regional nodes.

\section{Dosimetric outcomes}

Figure 1 shows a CT planning axial image through the same portion of the left breast in FB (Fig. 1a) and DIBH (Fig. 1b) in a patient with the IMC nodes included in the $80 \%$ breast prescription isodose line. Comparison of average dose parameters to target volumes and OARs for $\mathrm{FB}$ and DIBH are summarized in Table 2. The average $\mathrm{D}_{95}$ coverage of $\mathrm{CTV}_{\mathrm{b}}$ for the $\mathrm{B} / \mathrm{CWRT}+\mathrm{RNI}$ group in $\mathrm{FB}$ was slightly lower $(92.2 \pm 8.1 \%)$ than the planning dose constraint of $D_{95}>95 \%$. However, after correcting for multiple comparisons $\mathrm{D}_{95}$ of the $\mathrm{CTV}_{\mathrm{b}}$ was not significantly different between FB and DIBH for both cohorts. Patients in the $\mathrm{B} / \mathrm{CWRT}+\mathrm{RNI}$ group achieved comparable IMC coverage with $\mathrm{FB}$ and $\mathrm{DIBH}$ planning (average $\mathrm{V}_{80}$ for $\mathrm{CTV}_{\mathrm{IMC}}$ $99.7 \%$ and $98.9 \%$ for FB and DIBH respectively, $p=1.000$ ). Mean $\mathrm{CTV}_{\mathrm{b}}$ volume was comparable for FB vs. DIBH in both cohorts.

The DIBH technique significantly reduced $\mathrm{D}_{\text {mean }}$ Heart compared to FB for both the WBRT $(p=0.001)$ and B/ CWRT + RNI $(p=0.004)$ groups. DIBH also significantly reduced $\mathrm{D}_{\text {mean }} \mathrm{LAD}$ in both treatment cohorts $(p<0.005$ for both) when compared to FB. Heart $V_{25}$ was not significantly reduced with DIBH in either of the two cohorts alone; while the combined group of all patients showed a significant difference $(p=0.002)$. There was no significant difference between FB and DIBH in left lung $\mathrm{V}_{20}$ for either cohort alone or for all patients combined.

All patients in the WBRT group were able to meet a $D_{\text {mean }}$ Heart of $<4$ Gy on FB planning, but only five of nine patients (56\%) in the B/CWRT + RNI group were able to meet this constraint with FB. With DIBH, all patients in both cohorts were able to meet the $\mathrm{D}_{\text {mean }}$ Heart $<4$ Gy constraint.

Average relative percent reduction per patient in OAR dose parameters with DIBH are presented in Table 3. While Table 2 demonstrates that both groups of patients benefit from DIBH, the data presented in Table 3 shows that patients receiving regional nodal irradiation achieved a statistically significantly greater percent reduction in $D_{\text {mean }}$ Heart and LAD from DIBH then those receiving WBRT alone. The reduction in left lung $\mathrm{V}_{20}$ with DIBH was modest $(<10 \%)$ and not significantly different between WBRT and $\mathrm{B} / \mathrm{CWRT}+\mathrm{RNI}$ groups.

\section{Discussion}

There is a wealth of evidence from retrospective and planning studies demonstrating reduction in dose to the heart and coronary arteries with DIBH treatment of left-sided breast cancers. However, most published large retrospective 


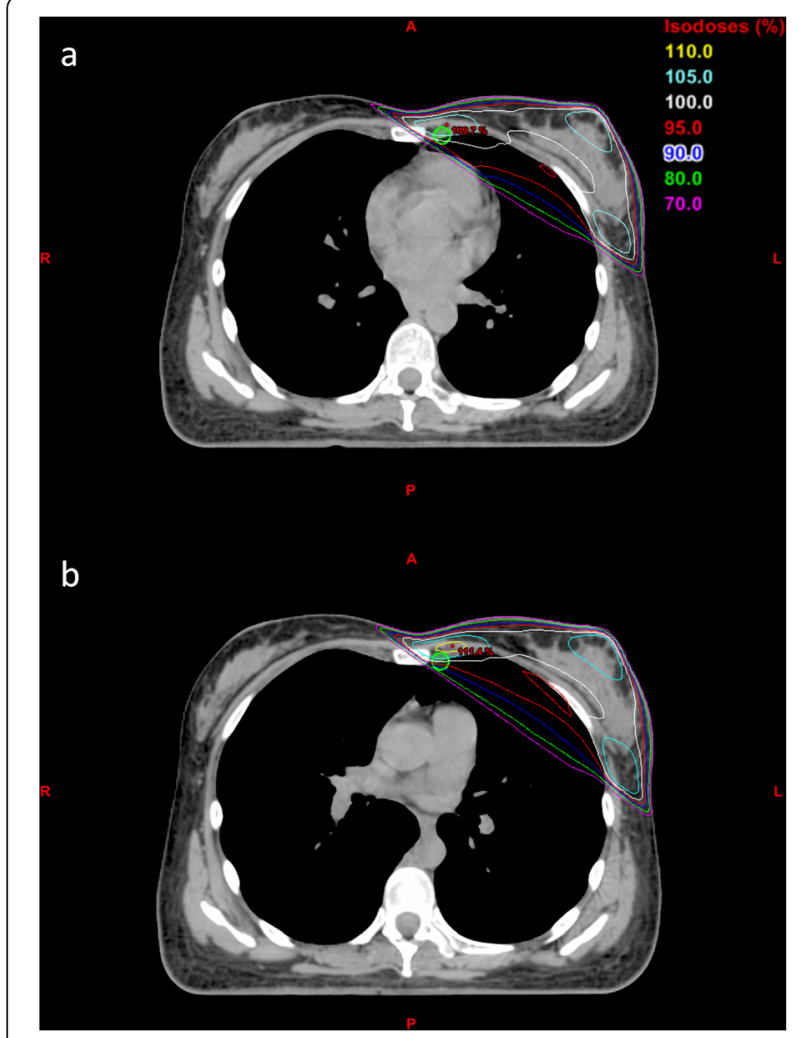

Fig. $1 C T$ simulation axial images of one patient at (a) FB and (b) $\mathrm{DIBH}$ with treatment plan isodose lines. The IMC nodes contour is shown in green

studies have combined all patients, irrespective of regional nodal irradiation in their analysis [8]. Similarly, few published planning studies have compared dosimetric outcomes between patients receiving WBRT alone (without IMC treatment) and those requiring additional regional nodal irradiation including treatment of the IMC nodes [7-14]. A planning study from Toronto demonstrated that DIBH reduced heart dose for some patients treated with wide breast tangents but included only five patients [15]. The current study is one of the larger studies evaluating differences in OAR dose reductions with DIBH comparing WBRT alone to patients receiving breast/chest wall plus regional nodal irradiation employing a modified wide tangents technique to include the IMC nodes.

In this study, the average $C T V_{b}$ volumes and $D_{95}$ coverage with DIBH were comparable to FB within each cohort, confirming minimal bias in regards to volume delineation and planning. The study results are similar to published retrospective and planning studies demonstrating that DIBH lowered $\mathrm{D}_{\text {mean }}$ Heart and LAD doses [7-15]. Planning studies have shown variable results regarding the impact of DIBH on ipsilateral lung dose-volume relationships. Some authors have reported that DIBH significantly reduced lung dose while others showed no difference [9-13].
In the current study, DIBH did not significantly reduce left lung $\mathrm{V}_{20}$. It is possible that the small sample sizes used in this study, however, did not achieve the statistical power to show this effect. Further studies with larger sample sizes are required to determine if there is a statistical difference in lung $\mathrm{V}_{20}$ between FB and DIBH.

DIBH significantly reduced both $\mathrm{D}_{\text {mean }}$ Heart and LAD doses in both treatment cohorts. A literature review of clinical and dose volume predictors for radiation-induced heart disease found no consensus regarding a safe threshold dose for the heart or LAD [22]. However, the ongoing NSABPB-51/RTOG1104 study protocol has recommended a $\mathrm{D}_{\text {mean }}$ Heart constraint for left sided breast/chest wall irradiation of $<4$ Gy [16]. Although there is evidence to suggest no safe threshold dose to the heart, the proposed dose of $<4$ Gy served as a reference to enable comparison in the current study $[4,23]$. All patients in the WBRT group were able to meet a $D_{\text {mean }}$ Heart < 4 Gy with $\mathrm{FB}$ while only slightly over half of the patients receiving regional nodal irradiation were able to meet this constraint with FB. All patients were able to meet this constraint with $\mathrm{DIBH}$, suggesting that $\mathrm{DIBH}$ provides a greater benefit for patients receiving regional nodal irradiation when using a modified wide tangent technique. Similarly, the relative percent reduction in $D_{\text {mean }}$ Heart and LAD dose with DIBH was significantly greater in the B/CWRT + RNI group compared to the WBRT group. To improve treatment planning efficiency, these observations have led to an institutional policy to use DIBH in all patients with left-sided breast cancer when the RT intent is to include the regional lymph nodes, but to use it selectively if the RT intent is whole breast RT alone.

In this study a modified wide tangent technique was used for IMC nodal irradiation. Results from some treatment planning studies have suggested that partially-wide tangent techniques may provide superior dosimetric coverage of the IMC nodes compared to photon-electron techniques $[24,25]$. The disadvantage of using modified wide tangents for IMC node irradiation is the potentially increased dose to the heart and lung; however we have shown here that DIBH significantly reduces this effect. The relative effectiveness of DIBH for patients receiving alternate forms of regional nodal irradiation, such as the photon-electron technique, is not examined in this study.

A limitation of the current study is the relatively small sample size. However, the patients were consecutive eligible cases, so the findings may more readily translate to other populations. Furthermore, all patients treated to the regional lymph nodes also had the IMC nodes included. Whether DIBH would be needed as frequently if the IMC nodes were excluded could not be addressed by the current data. However, the report of the EORTC study showing a survival advantage for the use of IMC plus medial supraclavicular RT may increase the frequency of IMC treatment [26]. Both $\mathrm{D}_{\text {mean }}$ Heart and $\mathrm{D}_{\text {mean }}$ LAD 
Table 2 Comparison of average dose parameters for targets and OARs for left-sided breast cancers by treatment cohort

\begin{tabular}{|c|c|c|c|c|c|c|}
\hline Parameter & WBRT & $\begin{array}{l}\text { WBRT FB vs. DIBH } \\
p \text {-value* }\end{array}$ & $\mathrm{B} / \mathrm{CWRT}+\mathrm{RNI}$ & $\begin{array}{l}\text { B/CWRT FB vs. DIBH } \\
p \text {-value }\end{array}$ & All Patients & $\begin{array}{l}\text { FB vs. DIBH } \\
p \text {-value* }\end{array}$ \\
\hline \multicolumn{7}{|c|}{$\mathrm{CTV}_{\mathrm{b}}$ Volume (cc) } \\
\hline FB & $515.5 \pm 356.9$ & 0.577 & $489.2 \pm 200.8$ & 0.496 & $503.7 \pm 290.2$ & 0.349 \\
\hline $\mathrm{DIBH}$ & $511.8 \pm 354.6$ & & $491.3 \pm 218.6$ & & $502.6 \pm 294.0$ & \\
\hline \multicolumn{7}{|c|}{$D_{95}$ for $C V_{b}(\%)$} \\
\hline $\mathrm{FB}$ & $97.3 \pm 1.4$ & 0.286 & $92.2 \pm 8.1$ & 0.030 & $95.0 \pm 5.9$ & 0.013 \\
\hline $\mathrm{DIBH}$ & $97.7 \pm 1.3$ & & $96.2 \pm 3.0$ & & $97.0 \pm 2.3$ & \\
\hline \multicolumn{7}{|l|}{$V_{80} I M C(\%)$} \\
\hline $\mathrm{FB}$ & N/A & N/A & $99.7 \pm 0.9$ & 1.000 & $\mathrm{~N} / \mathrm{A}$ & N/A \\
\hline $\mathrm{DIBH}$ & N/A & & $98.9 \pm 3.3$ & & N/A & \\
\hline \multicolumn{7}{|c|}{$D_{\text {mean }}$ Heart (cGy) } \\
\hline $\mathrm{FB}$ & $166.1 \pm 71.0$ & $0.001^{* *}$ & $375.7 \pm 186.0$ & $0.004^{* *}$ & $260.5 \pm 169.3$ & $<0.001^{* *}$ \\
\hline $\mathrm{DIBH}$ & $110.8 \pm 32.1$ & & $145.0 \pm 37.7$ & & $126.2 \pm 38.0$ & \\
\hline \multicolumn{7}{|c|}{$V_{25}$ Heart (\%) } \\
\hline $\mathrm{FB}$ & $0.8 \pm 1.4$ & 0.059 & $4.0 \pm 3.4$ & 0.014 & $2.2 \pm 2.9$ & $0.002^{* *}$ \\
\hline $\mathrm{DIBH}$ & $0.1 \pm 0.4$ & & $0.0 \pm 0.1$ & & $0.1 \pm 0.3$ & \\
\hline \multicolumn{7}{|c|}{$D_{\text {mean }} L A D(c G y)$} \\
\hline$F B$ & $1004.6 \pm 892.4$ & $0.002^{* *}$ & $1802.8 \pm 917.8$ & $0.004^{* *}$ & $1363.8 \pm 969.4$ & $<0.001^{* *}$ \\
\hline $\mathrm{DIBH}$ & $420.0 \pm 277.4$ & & $396.2 \pm 81.5$ & & $409.3 \pm 208.4$ & \\
\hline \multicolumn{7}{|c|}{$V_{20}$ Left Lung (\%) } \\
\hline $\mathrm{FB}$ & $10.5 \pm 4.6$ & 0.067 & $18.3 \pm 5.1$ & 0.250 & $14.0 \pm 6.2$ & 0.029 \\
\hline $\mathrm{DIBH}$ & $8.5 \pm 3.6$ & & $16.3 \pm 2.8$ & & $12.0 \pm 5.1$ & \\
\hline \multicolumn{7}{|c|}{ Patients meeting } \\
\hline \multicolumn{7}{|c|}{$\mathrm{D}_{\text {mean }}<4 \mathrm{~Gy}$ (fraction, \%) } \\
\hline FB & $11 / 11(100)$ & $\mathrm{N} / \mathrm{A}$ & $5 / 9(56)$ & 0.134 & $16 / 20(80)$ & 0.134 \\
\hline $\mathrm{DIBH}$ & $11 / 11(100)$ & & 9/9 (100) & & $20 / 20(100)$ & \\
\hline
\end{tabular}

Wilcoxon signed-rank test is used for comparison of all continuous variables, and McNemar's test is applied for discrete variables Significant $p$-values after adjusting for multiple testing are indicated by a double asterisk $(* *)$

Abbreviations: WBRT $=$ whole breast $\mathrm{RT} \pm$ boost cohort, $\mathrm{B} / \mathrm{CWRT}+\mathrm{RNI}=$ breast or chest wall + regional nodal $\mathrm{RT}$ cohort, $\mathrm{FB}=\mathrm{free}-\mathrm{breathing}, \mathrm{DIBH}=\mathrm{deep}$ inspiration breath hold, $C T V_{b}=C T V$ for breast or chest wall, IMC $=$ internal mammary chain nodes, $D_{95}=$ Dose to $95 \%$ target volume, $V_{80}=\%$ volume of organ receiving $80 \%$ of the prescription dose, $V_{25}=\%$ volume of organ receiving $25 \mathrm{~Gy}, \mathrm{D}_{\text {mean }}=$ Mean dose, LAD = left anterior descending coronary artery, $\mathrm{V}_{20}=\%$ volume of organ receiving $20 \mathrm{~Gy}, \mathrm{~N} / \mathrm{A}=$ not applicable. Data shown are mean values with one standard deviation. *In this group, to achieve significance $\mathrm{p}$-values must be $<0.0083$ to account for multiple testing. ${ }^{* *}$ In this group, to achieve significance $p$-values must be $<0.0071$ to account for multiple testing. ${ }^{* *}$ Indicates significance when compared to the Bonferroni adjusted criterion

Table 3 Average patient percent relative reduction in dose parameters with DIBH compared to FB for left-sided breast cancer patients by treatment cohort. Significant $p$-values after adjusting for multiple testing are indicated by a double asterisk

\begin{tabular}{llll}
$\left(*^{*}\right)$ & & & \\
\hline Parameter & WBRT & B/CWRT + RNI & $p$-value* \\
\hline$D_{\text {mean Heart }}$ & $29.2 \%$ & $55.9 \%$ & $0.003^{* *}$ \\
D $_{\text {mean }}$ LAD & $43.5 \%$ & $72.1 \%$ & $0.014^{* *}$ \\
$V_{20}$ Left Lung & $8.9 \%$ & $6.6 \%$ & 0.305 \\
\hline
\end{tabular}

Wilcoxon sum-rank test used in for comparison of all variables *In this group, to achieve significance p-values must be $<0.0167$ to account for multiple testing. ${ }^{* *}$ Indicates significance when compared to the Bonferroni adjusted criterion were significantly reduced in both groups. One may argue that the LAD is a more important target to avoid in the pathogenesis of long term cardiac complications [14, 27]. Further studies are required to determine threshold doses to cardiac structures in breast radiotherapy.

\section{Conclusions}

DIBH provided greater percent reductions in mean heart and LAD doses for patients receiving regional nodal irradiation that included the IMC nodes using a modified wide tangent technique than for whole breast RT alone. All patients receiving WBRT alone met the mean heart dose constraint of $<4$ Gy on free breathing planning, while only slightly over half of patients receiving regional nodal irradiation were able to meet this constraint in free breathing. 
DIBH is justified for all patients receiving RT for left-sided breast cancer, but as a minimum, should be used regularly for all left-sided breast cancer patients receiving breast/ chest wall RT plus nodal RT.

\section{Competing interests}

The authors declare that they have no competing interests.

\section{Authors' contributions}

$R Y, K L, D W, W S, A H, T P$ designed and coordinated the study. RY and LC performed the data analysis. $\mathrm{HL}$ and $\mathrm{LC}$ performed the statistical analysis. RY and TP performed the interpretation of the data. RY, LC, and TP drafted the manuscript. All authors read and approved the final manuscript.

\section{Author details}

'Division of Radiation Oncology, Department of Oncology, University of Calgary, 2500 University Dr NW, Calgary, Alberta T2N 1 N4, Canada. ${ }^{2}$ Department of Medical Physics, Tom Baker Cancer Centre, 133129 Street Northwest, Calgary, AB T2N 4 N2, Canada. ${ }^{3}$ Department of Physics \& Astronomy, University of Calgary, 2500 University Dr NW, Calgary, Alberta T2N 1 N4, Canada. ${ }^{4}$ Department of Radiation Therapy, Tom Baker Cancer Centre, 133129 Street Northwest, Calgary, AB T2N 4 N2, Canada. ${ }^{5}$ Departments of Oncology and Community Health Sciences, University of Calgary, 2500 University Dr NW, Calgary, Alberta T2N 1 N4, Canada. ${ }^{6}$ Division of Medical Physics, Department of Oncology, University of Calgary, 2500 University Dr NW, Calgary, Alberta T2N 1 N4, Canada.

Received: 8 June 2015 Accepted: 15 September 2015

Published online: 22 September 2015

\section{References}

1. Clarke M, Collins R, Darby S, Davies C, Elphinstone P, Evans V, et al. Effects of radiotherapy and of differences in the extent of surgery for early breast cancer on local recurrence and

15-year survival: an overview of the randomized trials. Lancet. 2005;366:2087-106.

2. EBCTCG (Early Breast Cancer Trialists' Collaborative Group), McGale P, Taylor C, Correa C, Cutter D, Duana F, et al. Effect of radiotherapy after mastectomy and axillary surgery on

10-year recurrence and 20-year breast cancer mortality: meta-analysis of individual patient data for 8135 women in 22 randomised trials. Lancet. 2014;383:2127-35.

3. Paszat LF, Mackillop WJ, Groome PA, Schulze K, Holowaty E. Mortality from myocardial infarction following post-lumpectomy radiotherapy for breast cancer: a population-based study in Ontario, Canada. Int J Radiat Oncol Biol Phys. 1999:43:755-62.

4. Darby SC, Ewertz M, McGale P, Bennet AM, Blom-Goldman U, Bronnum D, et al. Risk of ischemic heart disease in women after radiotherapy for breast cancer. N Eng J Med. 2013;368:987-98.

5. Giordano SH, Kuo YF, Freeman JL, Buchholz TA, Hortobaqyi GN, Goodwin JS. Risk of cardiac death after adjuvant radiotherapy for breast cancer. J Natl Cancer Inst. 2005;97:419-24.

6. Shapiro CL, Hardernbergh PH, Gelman R, Blanks D, Hauptman P, Recht A, et al. Cardiac effects of adjuvant doxorubicin and radiation therapy in breast cancer patients. J Clin Oncol. 1998;16:3493-501.

7. Hjelstuen MHB, Mjaaland I, Vikström J, Dybvik KI. Radiation during deep inspiration allows loco-regional treatment of left breast and axillary-, supraclavicular and internal mammary lymph nodes without compromising target coverage or dose restrictions to organs at risk. Acta Oncol. 2012;51:33344.

8. Nissen HD, Appelt AL. Improved heart, lung, and target dose with deep inspiration breath hold in a large clinical series of breast cancer patients. Radiother Oncol. 2013;106:28-32.

9. Korreman SS, Pedersen AN, Nøttrup TJ, Specht L, Nyström H. Breathing adapted radiotherapy for breast cancer: comparison of free breathing gating with the breath-hold technique. Radiother Oncol. 2005;76:311-8.

10. Pedersen AN, Korreman S, Nyström H, Specht L. Breathing adapted radiotherapy of breast cancer: reduction of cardiac and pulmonary doses using voluntary inspiration breath hold. Radiother Oncol. 2004;72:53-60.
11. Remouchamps VM, Vicini FA, Sharpe MB, Kestin LL, Martinez AA, Wong JW. Significant reductions in heart and lung doses using deep inspiration breath hold with active breathing control and intensity modulated radiation therapy for patients treated with locoregional breast irradiation. Int J Radiat Oncol Biol Phys. 2003;55:392-406.

12. Hayden A, Rains M, Tiver K. Deep inspiration breath hold technique reduces heart dose from radiotherapy for left-sided breast cancer with deep breath-holding. J Med Imaging Radiat Oncol. 2012;56:464-72.

13. Nemoto K, Ogushi M, Nakajima M, Kozuka T, Nose T, Yamashita T. Cardiacsparing radiotherapy for left breast cancer with deep breath-holding. Jpn J Radiol. 2009:27:259-63.

14. Lu HM, Cash E, Chen MH, Chin L, Manning WJ, Harris J, et al. Reduction of cardiac volume in left-breast treatment field by respiratory maneuvers: a CT study. Int J Radiat Oncol Biol Phys. 2000;47:895-904.

15. Sixel K, Aznar M, Ung Y. Deep inspiration breath hold to reduce irradiated heart volume in breast cancer patients. Int J Radiat Oncol Biol Phys. 2001:49:199-204.

16. Breast NSA, Project B. NSABP Protocol B-51: A randomized phase III clinical trial evaluating post-mastectomy chestwall and regional nodal xrt and post-lumpectomy regional nodal xrt in patients with positive axillary nodes before neoadjuvant chemotherapy who convert to pathologically negative axillary nodes after neoadjuvant chemotherapy. Pittsburgh: NSABP; 2013 [Available at: mtcancer.org/Protocols/B51_Protocol.pdf; cited April 25, 2014].

17. Sadaro A, Petruzzelli MF, D'Errico MP, Grimauldi L, Pili G, Portaluri M. Radiation-induced cardiac damage in early left breast cancer patients: risk factors, biological mechanisms, radiobiology, and dosimetric constraints. Radiother Oncol. 2012;103:133-42

18. Nilsson G, Holmberg L, Carmo H, Duvernoy O, Sjögren I, Lagerqvist B, et al, Distribution of coronary artery stenosis after radiation for breast cancer. J Clin Oncol. 2012;30:380-6.

19. White J, Tai A, Arthur D, Buchholz T, MacDonald S, Marks L, et al. Radiotherapy Oncology Group breast cancer atlas for radiation therapy planning: consensus definitions. [Available at: http://www.rtog.org/ LinkClick.aspx?fileticket=vzJFhPaBipE\%3d\&tabid=23; cited June 15, 2013].

20. Marks LB, Hebert ME, Bentel G, Spencer DP, Sherouse GW, Prosnitz LR. To treat or not to treat the internal mammary nodes: a possible compromise. Int J RadiatOncolBiolPhys. 1994;29:903-9.

21. Feng M, Moran JM, Koelling T, Chughtai A, Chan JL, Freedman L, et al. Development and validation of a heart atlas to study cardiac exposure to radiation following treatment for breast cancer. Int J Radiat Oncol Biol Phys. 2011;79:10-8.

22. R Core Team (2015). R: A language and environment for statistical computing. R Foundation for Statistical Computing. Vienna, Austria. http:// www.R-project.org. Accessed 01 Sept 2015.

23. Gagliardi G, Constine LS, Moiseenko V, Correa C, Pierce LJ, Allen AM, et al. Radiation Dose-Volume Effects in the Heart. Int J Radiat Oncol Biol Phys. 2010;76(3 Suppl):S77-85.

24. Arthur DW, Arnfield MR, Warwicke LA, Morris MM, Zwicker RD. Internal mammary node coverage: an investigation of presently accepted techniques. Int J Radiat Oncol Biol Phys. 2000;48:139-46.

25. Severin D, Connors S, Thompson H, Rathee S, Stavrev P, Hanson J. Breast radiotherapy with inclusion of internal mammary nodes: a comparison of techniques with three-dimensional planning. Int J Radiat Oncol Biol Phys. 2003;55:633-44.

26. Poortmans P, Struikmans H, Kirkove C, Budach V, Malngon P, Valli MC et al. Irradiation of the internal mammary and medial supraclavicular lymph nodes in stage I to III breast cancer: 10 years results of the EORTC Radiation Oncology and Breast Cancer Groups phase III trial 22922/10925 [Abstract]. Eur J Cancer. 2013;47:2.

27. Konings AW, Smit Sibinga CT, Aarnoudse MW. Initial events in radiation induced atheromatosis. Damage to intimal cells. Strahlen therapie. 1978;154:795-800. 